\title{
Sustaining essential TB services in Sri Lanka amid COVID-19 pandemic: a brief report
}

\author{
Mizaya Cader," Nirupa Pallewatte, Dushani Jayawardhana, Sumudu Avanthi Hewage, Lakmal \\ Rathnayaka
}

National Programme for Tuberculosis Control and Chest Diseases, Ministry of Health, Sri Lanka

"Correspondence: mizaya1971@gmail.com

iDhttps://orcid.org/0000-0002-4088-5355

DOI: https://doi.org/10.4038/jccpsl.v26i5.8364

Received on 22 May 2020

Accepted on 9 June 2020

\section{Summary}

National Programme for Tuberculosis Control and Chest Diseases (NPTCCD) functions via a network of district chest clinics (DCCs) located in all 26 districts. Further diagnostic and treatment services are provided via 108 branch clinics and more than 160 microscopic centres in decentralized institutions located island wide to deliver accessible diagnostic and treatment services for the catering community (1).

The spread of COVID-19 in Sri Lanka and subsequent preventive measures launched by the government, including lock down situations have imposed a potential threat to TB case finding and effective management of already diagnosed TB patients. However, considering the importance of ensuring the essential services and operation for long-standing health problems like tuberculosis, NPTCCD has taken many efforts to ensure provision of uninterrupted quality TB services at district level (2). Sri Lanka, being one of the countries that has committed to reach the End-TB targets by the year 2025 with the aim of reducing TB incidence and fatalities in a significant manner (3), we understood that it is utmost important to cater TB services at our best at this juncture. At the same time, we realized the importance of strengthening preventive measures in the community not only to prevent the spread of TB but also to avoid emergence of TB-COVID-19 comorbidities as latter situation will usually ends up in unfavourable TB outcomes (2). As the focal point, we faced the dual challenge of sustaining TB care while safeguarding the health care staff who are directly in contact with the presumptive TB patients who may also be potential COVID-19 patients, as all institutions that provide TB care encounter patients with respiratory symptoms such as cough, fever and difficulty in breathing common to both TB and COVID-19 (4). This narrative report highlights the key actions taken by the NPTCCD to sustain essential TB services at district level while providing guidance on safety precautions for the curative and preventive staff. 


\section{Public health response and its impact}

To streamline tuberculosis (TB) diagnostic and treatment services as well as infection prevention and control (IPC) measures in these institutions, directives were provided via guidelines addressing the following aspects. NPTCCD issued two guidelines, namely the Guidelines on Diagnosis and Management of TB Patients at District Level During Current COVID-19 Pandemic Situation (4) and Interim Guidance for Intermediate TB Laboratories, Genexpert Laboratories and Microscopy Centres for handling patients' samples amid COVID-19 pandemic (5). This brief report elaborates on how we sustained TB service provision amid Covid19 pandemic in the DCC settings, decentralized institutions and in the community.

\section{- TB Diagnostic services at DCCs and decentralized institutions}

CCs continued provision of minimum essential services for TB patients. Since TB patients are at a higher risk of ending up in poor treatment outcomes when infected with COVID-19, action was taken to minimize the routine clinic attendance of already diagnoed TB patients. If any attendees were found, they were segregated to minimize cross-infections and attended without delay. However, attendance of new patients with acute respiratory symptoms was not encouraged at the DCCS. If any new clinic attendees withrespiratory symptoms were found, these patients were triaged and patients with acute respiratory symptoms were filtered out to fast track the investigation procedures (6). Further, instructions were provided for the DCC laboratories to provide relevant sevices without delay if a patient visits the DCC for sputum examination (sputum AFB), either for diagnosis or follow up. Similarly, if any patient needs to undergo a TB rapid diagnostic test (GeneXpert), the samples were sent to the respective institutionswhere GeneXpert machines are located. In order to facilitate this, all 31 GeneXpert machines located in 26 districts were utilized for service provision. Although utilization of GeneXpert machines under NPTCCD was proposed for COVID19 diagnosis, it was nt implemented. Hence, TB diagnostic services provided through GeneXpert were not hampered. Further, uninterrupted TB culture services were provided via national TB reference laboratory (NTRL) and four intermediate TB laboratories (ITLs) located in Kandy, affna, Galle and Rathnapura Districts. Radiological facilities were provided for the patients who had strong indications for, as per to facilitate clinical diagnosis and other situations indicated by the consultant respiratory physicians (4).

As per the instructions, microscopic centres at decentralized institutions continued provision of diagnostic services for inward and outpatient presumptive TB patients. However, according to the human resource availability and risk category of the localityfor COVID-19, some institutions practised collection and storage of sputum. Examination of the collected specimens was carried out on selected days of the week (4).

\section{- TB treatment services at DCCs and decentralized institutions}

All measures were taken to ensure supply of drugs for already diagnosed TB patients. Drugs were supplied for an extended duration considering the treatment phase of each TB patient. Two weeks supply of drugs was provided for the patients who were in the inensive phase while one-month supply of drugs was provided for those who have completed the intensive phase of treatment. If any patient was completing treatment in due course and not attended for radiological and clinical assessment, an extended period oftwo weeks supply of drugs was provided until proper assessment. Arrangements were made to deliver all these drugs either directly to the residence of the patient, or to a directly observed treatment (DOT) centre utilizing DCC vehicle. Public health inspecors (PHI) attached to the DCC extended their support in the field. The DCC staff worked as a team to ensure provision of drugs from packaging up to delivery. When the drugs were delivered to a place other than the residence of the patient (DOT centre), ths was reconfirmed by contacting each patient over the phone. If clinical monitoring of a patient was needed as in situations like bridging therapy or extra pulmonary TB patients, they were asked to present physically. Continuous monitoring of the patientson 
drug intake and side effects was carried out over the phone (4).

Facilities were provided for all the decentralized institutions to initiate treatment without referring the patients to DCCs once the diagnosis is made either as inward or as outpatient. To enable this, a buffer stock of drugs adequate for at least 1-5 patents was made available in larger decentralized institutions. Drugs were made available with the chief pharmacist of the institution and kept under optimal condition. At central level, the central drug stores ensured provision of adequate supply of stocksto all the districts.

\section{- Infection prevention and control measures at DCCs and decentralized institutions}

An interim guidance for intermediate TB laboratories, Genexpert laboratories and microscopy centres for handling patients' samples was developed together with the consultant microbiologist. The guide highlighted that people ill with COVID-19 show symptoms imilar to TB and therefore exerts an additional risk when managing these patients and handling samples in TB laboratories. Infection control measures for sample collection, transport, reception of samples and sample processing were specified in the guidelnes. Further, general measures for all the laboratories such as environmental control measures, hand hygiene, staff distancing were explained. Instructions were given to use biosafety cabinets when handling sputum and any other infectious specimen during he pandemic. Considering the risk level of each laboratory setting, the type of personal protective equipment (PPE) that should be used was specified. As the staff in TB culture laboratories engage in aerosol generating procedures, specific instructions wre given on the use of N95 mask, gloves (double), gown and goggles or protection shield and waterproof aprons before processing samples (5-8). As DCCs are not conducting any aerosol generating procedures and majority of the clinics have biosafety cabinets the recommendation was given to use surgical masks together with other PPEs. The staff working in the microscopic centres in decentralized institutions located in high COVID-19 risk districts were provided N95 masks with other PPEs. A special informationguide was circulated on how to don (wear), use, and doff (remove) a face mask during day to day activities. Considering infectious waste management as a priority area in this pandemic situation, proper guidance on infectious waste management in DCCs, ITLsand microscopic centres was provided (5-8).

- Community awareness through social media campaigns

The COVID-19 pandemic created a situation that the district team was not in a position to conduct advocacy and awareness by meeting the stakeholders and the community. Hence, the available social media platform was utilized for effective communication to pevent the spread of TB and to prevent COVID-19 among already diagnosed TB patients. We believed building the trust in our services among already diagnosed TB patients who were on treatment was paramount at that time point, in order to improve the treatmen adherence of already diagnosed TB patients. Hence, a focal point was identified in each district and the hotline numbers were circulated through social media. This enabled provision of any information on TB disease, COVID19 , and preventive, diagnostic ad treatment services available for the patients and the community during the pandemic. Information on delivery of drugs to the residences of the patients, instructions about treatment adherence and general respiratory precautions were frequently circulate via NPTCCD social media page. We took the opportunity to educate people on the importance of TB contact screening as COVID-19 preventive strategy followed the same principle. IEC materials were developed and circulated to educate the patients on infectius nature of both the diseases, highlighting tuberculosis is yet another infectious disease that can spread to any person, with the aim to reduce the stigma and discrimination attached to TB.

\section{Conclusions}

As the focal point of TB control in Sri Lanka, we made our best possible effort to sustain the hard work we have done over the years for the prevention and 
control of tuberculosis amid this COVID-19 pandemic. Many challenges were addressed effectively and fficiently to provide essential minimum care for diagnosis and treatment while safeguarding the lives of service providers by applying suitable IPC measures and preventing TBCOVID-19 comorbidities by launching awareness campaigns via social media platfors. Management of COVID-19 pandemic in Sri Lanka did not make TB services over stretched when compared to many other countries where majority of the services had been diverted to COVID-19 service provision. Thus, the Ministry of Health has made a tremendou contribution for smooth functioning of TB services, supporting the integrated effort of ending the TB epidemic in Sri Lanka by the year 2025.

\section{Author Declaration}

Acknowledgements: We acknowledge all the district tuberculosis control officers and their teams for their dedication in carrying out TB control activities at district level and Dr Amitha Fernando, consultant respiratory physician for his contribution in developing guidelines.

Author contributions: MC, NP, DJ, SH and LR contributed for developing the guidelines and providing instructions for the districts for implementation of the specified actions. MC led, and all the other authors contributed to the drafting and revision of the report.

\section{Reference}

1. National Programme for Tuberculosis Control and Chest Disease. National Strategic Plan for Tuberculosis control 2015-2020. Colombo: Ministry of Health, Sri Lanka. Available from: http://www. nptccd.info/wpcontent/uploads/2019/11/NSP-SriLanka.

2. Ministry of Health, Sri Lanka. Ending Tuberculosis and AIDS in Sri Lanka: Urgent and immediate actions required to reach 2025 targets. Available from: http://www. nptccd. info/wp-content/ uploads/2019/03/Ending-TB-AIDS.

3. World Health Organisation. COVID-19: considerations for tuberculosis (TB) care services. Available from: https://www.who.int /docs/defaultsource/documents /tuberculosis/ infonote-tb-covid19.pdf. Access 20 May 2020.

4. National Programme for Tuberculosis Control and Chest Disease, Ministry of Health, Sri Lanka. Guidelines on Diagnosis and Management of TB Patients at District Level During Current COVID-19 Pandemic Situation. Available from: https://www. nptccd.info/wp-content/uploads/ 2020/03/Circularcovid-19-TB-new.pdf.

5. National Programme for Tuberculosis Control and Chest Disease, Ministry of Health, Sri Lanka. Interim Guidance for Intermediate TB Laboratories, Genexpert Laboratories and Microscopy Centres for Handling Patients' Samples amid COVID-19 Pandemic. Available from: https://www. nptccd.info/ wp-content/uploads/ 2020/04/Circular-covid-19-TBnew.pdf

6. Centres of Disease Control. Interim Infection Prevention and Control Recommendations for Patients with Suspected or Confirmed Coronavirus Disease 2019 (COVID-19) in Healthcare Settings. Available from: https://www.cdc.gov/ corona virus/2019-ncov/hcp/dialysis.html.

7. World Health Organisation. Guidelines Infection Prevention and Control of Epidemic-and Pandemicprone Acute Respiratory Infections in Health Care. Available from: https://www.who.int/csr/ biorisk reduction/infection_control/publication/en.

8. World Health Organization. Infection prevention and control during health care when novel coronavirus ( $n \mathrm{CoV})$ infection is suspected. Available from: https://www.who.int/ publications-detail/ infectionprevention-and-control-during-health-care-whennovel-coronavirus-(ncov)-infection-is-suspected20200125. 